\title{
Patogenicidade de Beauveria bassiana ao psilídeo Diaphorina citri e compatibilidade do fungo com produtos fitossanitários
}

\author{
Ana Paula Ferreira Pinto(1), Antonio Batista Filho(1), José Eduardo Marcondes de Almeida(1) \\ e Inajá Marchizeli Wenzel(1)
}

(1)Instituto Biológico, Laboratório de Controle Biológico, Rodovia Heitor Penteado, Km 3, Caixa Postal 70, CEP 13001-970 Campinas, SP.
E-mail: aninhafp@gmail.com, batistaf@biologico.sp.gov.br, jemalmeida@biologico.sp.gov.br, iawenzel@yahoo.com.br

Resumo - O objetivo deste trabalho foi avaliar a patogenicidade de Beauveria bassiana a ninfas de Diaphorina citri (Hemiptera: Psyllidae) e verificar a compatibilidade do fungo com produtos fitossanitários e sua persistência em plantas de citros. Ninfas de D. citri foram pulverizadas com B. bassiana, nas concentrações $5 \times 10^{6}, 1 \times 10^{7}, 5 \times 10^{7}, 1 \times 10^{8}, 5 \times 10^{8}$ e $1 \times 10^{9}$ conídios $\mathrm{mL}^{-1}$, para determinação da concentração letal. Para avaliação da compatibilidade do fungo com produtos fitossanitários, extrato de nim e cinco inseticidas de quatro grupos químicos diferentes foram incorporados individualmente ao meio de cultura BDA em que o fungo foi cultivado. Avaliaram-se o crescimento vegetativo, a esporulação e a viabilidade do entomopatógeno. Plantas de citros, mantidas em casa de vegetação, foram tratadas primeiramente com os produtos fitossanitários e depois com o entomopatógeno. Avaliaram-se os tempos de exposição de 24 horas e de 7 e 14 dias. O fungo

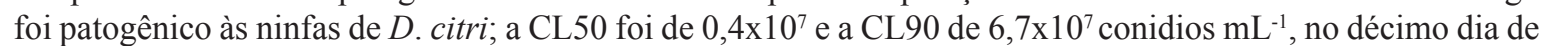
avaliação. Em laboratório, os produtos fitosssanitários reduzem o crescimento do fungo. Em casa de vegetação, os produtos não afetam a sobrevivência do fungo nas plantas de citros.

Termos para indexação: controle biológico, fungos entomopatogênicos, greening, "huanglongbing", psilídeo-dos-citros.

\section{Beauveria bassiana pathogenicity to Diaphorina citri and compatibility of the fungus with phytosanitary products}

\begin{abstract}
The objective of this work was to evaluate the pathogenicity of Beauveria bassiana to Diaphorina citri (Hemiptera: Psyllidae) nymphs, and to check the compatibility of the fungus with phytosanitary products, and its persistence in citrus plants. Nymphs of $D$. citri were sprayed with B. bassiana in the concentrations $5 \times 10^{6}, 1 \times 10^{7}, 5 \times 10^{7}, 1 \times 10^{8}, 5 \times 10^{8}$ and $1 \times 10^{9}$ conidia $\mathrm{mL}^{-1}$ for lethal concentration determination. In order to evaluate the compatibility of the phytosanitary products with the fungus, neem extract and five insecticides of four different chemical groups were individually added to PDA culture medium, in which the fungus was cultivated. Vegetative growth, sporulation and viability of the entomopathogen were evaluated. In the greenhouse, citrus plants were first treated with the phytosanitary products and, then, with the pathogen. Exposure times of 24 hours, 7 and 14 days were evaluated. Fungus pathogenicity to $D$. citri nymphs was confirmed; LC50 of $0.4 \times 10^{7}$ and LC90 of $6.7 \times 10^{7}$ conidia $\mathrm{mL}^{-1}$ were determined at the tenth day of evaluation. In the laboratory, the phytosanitary products reduced fungus growth. In greenhouse, the products did not affect fungus survival on citrus plants.
\end{abstract}

Index terms: biological control, entomopathogenic fungi, greening, huanglongbing, citrus psyllid.

\section{Introdução}

Dada a importância econômica da citricultura para o Brasil, espera-se que problemas fitossanitários recebam a devida atenção da pesquisa, pois pragas e doenças exóticas introduzidas no Brasil durante as últimas décadas têm causado severas perdas econômicas aos produtores (Nava et al., 2007).

Entre as doenças presentes na cultura está o huanglongbing (HLB), popularmente conhecido como “greening” (Tsai \& Liu, 2000; Alemán et al., 2007; Boina et al., 2009). Essa doença, atualmente, é a maior responsável por perdas na citricultura nacional. Até 2004, havia registros da doença somente nos continentes asiático e africano. A partir dessa data, foi registrada sua ocorrência no Estado de São Paulo (onde o primeiro relato é datado de março de 2004) e na Flórida (Bové, 2006; Alemán et al., 2007; Nava et al., 2007). 
A estratégia de controle mais utilizada atualmente consiste no controle do vetor da doença, Diaphorina citri Kuwayama (Hemiptera: Psyllidae) (Cocco \& Hoy, 2008; Avery et al., 2009; Sanches et al., 2009). Entre as principais técnicas de controle do inseto, está o controle químico, considerado como ferramenta importante para reduzir a disseminação e transmissão dos patógenos (Yamamoto et al., 2009). Contudo, o uso intensivo de agrotóxicos tem sérias restrições, pela contaminação do meio ambiente e danos à saúde humana e pelo efeito que têm em organismos não alvos, como insetos benéficos e animais silvestres e de criação. Assim, o controle biológico é uma alternativa que permite reduzir os danos causados pelo inseto-vetor, sem causar impactos negativos ao ambiente (Souza \& Reis, 1986; Pereira et al., 2004).

Apesar de D. citri ter sido constatada há mais de meio século nos pomares de citros do Brasil, trabalhos de controle dessa praga, com fungos entomopatogênicos, são escassos.

O objetivo deste trabalho foi avaliar a patogenicidade de Beauveria bassiana a ninfas de Diaphorina citri (Hemiptera: Psyllidae) e verificar a compatibilidade do fungo com produtos fitossanitários e sua persistência em plantas de citros.

\section{Material e Métodos}

Os ensaios foram desenvolvidos no Laboratório de Controle Biológico, do Centro Experimental Central do Instituto Biológico, sediado em Campinas, SP, de janeiro de 2011 a fevereiro de 2012.

Os insetos foram fornecidos pelo Departamento de Entomologia e Acarologia, Laboratório de Biologia de Insetos, da Escola Superior de Agricultura Luiz de Queiroz. A criação em laboratório seguiu a metodologia descrita por Gómez-Torres (2009), com plantas de murta (Murraya paniculata), com 25 a $30 \mathrm{~cm}$ de altura, cultivadas em substrato de vermiculita e composto vegetal (1:1).

Após podas sucessivas, as plantas com brotações foram transferidas para gaiolas de criação, confeccionadas com acrílico e tela antiafídeo $(60 \times 60 \times 50 \mathrm{~cm})$, que continham adultos de D. citri, para obtenção dos ovos. Não foi verificada a razão sexual. Após a oviposição, oito plantas com ovos foram transferidas para gaiolas de criação teladas
$(43 \times 43 \times 37,5 \mathrm{~cm})$, em sala climatizada $\left(25 \pm 1^{\circ} \mathrm{C}\right)$, para o desenvolvimento ninfal.

O isolado IBCB 66, do fungo entomopatogênico $B$. bassiana, foi obtido da Coleção de Fungos Entomopatogênicos Oldemar Cardim Abreu, do Laboratório de Controle Biológico do Centro Experimental Central do Instituto Biológico, localizado em Campinas, SP. O isolado foi armazenado em freezer a $-4^{\circ} \mathrm{C}$, na forma de conídios puros, em tubos de cultura com óleo mineral, e cultivado em placas com meio batata-dextrose-ágar (BDA), incubadas em câmara de germinação do tipo $\mathrm{BOD}$, a $25 \pm 1^{\circ} \mathrm{C}$ e fotófase de 12 horas, por 14 dias.

Para os testes de patogenicidade de B. bassiana a ninfas de $D$. citri e determinação das concentrações (conídios $\mathrm{mL}^{-1}$ ) letais de 50 e 90\% (CL50 e CL90) e do tempo letal médio 50 e 90 (dias), suspensões com $5 \times 10^{6}$, $1 \times 10^{7}, 5 \times 10^{7}, 1 \times 10^{8}, 5 \times 10^{8}$ e $1 \times 10^{9}$ conídios $\mathrm{mL}^{-1}$ foram preparadas a partir de placas de Petri que continham o isolado IBCB 66 do fungo.

A viabilidade dos conídios foi determinada pela técnica de microcultivo e exame direto em lâminas de microscopia (Marques et al., 2004). Só foram utilizadas suspensões que apresentaram viabilidade acima de $90 \%$.

Ninfas de D. citri, de segundo a quarto instar, foram mantidas em plantas de murta (M. paniculata) cultivadas em tubetes. Cada tubete foi considerado uma repetição, tendo-se avaliado cinco repetições por tratamento. A quantidade de ninfas por repetição não foi constante, em razão do número variado de ovos por brotação/repetição, tendo-se mantido sempre mais do que 110 ninfas por repetição. A inoculação dos conídios nas ninfas de $D$. citri foi realizada pela pulverização de $2 \mathrm{~mL}$ por tubete, de cada uma das suspensões fúngicas, em torre de Potter. As ninfas do tratamento testemunha foram pulverizadas com água destilada estéril e espalhante adesivo (Tween 80 ) a $0,1 \%$. As plantas foram mantidas em sala climatizada $\left(25 \pm 1^{\circ} \mathrm{C}\right.$ e fotófase de 14 horas), em gaiolas individuais confeccionadas com copos de plástico de $500 \mathrm{~mL}$ cobertos com tecido voil.

A sobrevivência das ninfas foi avaliada diariamente, até 10 dias após a pulverização dos conídios, e os insetos mortos foram transferidos para placas de Petri com algodão úmido (câmara úmida), e colocados em câmara de germinação tipo $\mathrm{BOD}$, a $25 \pm 1^{\circ} \mathrm{C}$ e 
fotófase de 12 horas, por 15 dias, para confirmação da mortalidade causada pelo patógeno.

Para determinação da compatibilidade de B. bassiana com produtos fitossanitários comumente utilizados na citricultura, foram avaliados em condições de laboratório cinco inseticidas e um extrato vegetal (Tabela 1). Os seguintes parâmetros foram quantificados: crescimento vegetativo, conidiogênese e viabilidade do fungo.

Os produtos foram adicionados ao meio de cultura BDA, em suas concentrações recomendadas para a cultura. O meio foi vertido em placas plásticas de Petri descartáveis, de 90x15 mm, e a inoculação dos fungos foi realizada após a solidificação do BDA.

Foram utilizadas cinco placas por tratamento, e a inoculação foi realizada em três pontos equidistantes da placa de Petri. A testemunha foi representada pelo meio de cultura sem a adição dos produtos fitossanitários. As placas foram mantidas em câmaras tipo BOD, a $25 \pm 1^{\circ} \mathrm{C}$, por sete dias e, após esse período, foi realizada a medição em dois sentidos perpendiculares, tendo-se obtido o diâmetro médio das colônias para avaliação do crescimento vegetativo. Para avaliação da conidiogênese, as colônias foram retiradas das placas juntamente com o meio de cultura, com o auxílio de um bisturi, e transferidas para tubos de ensaio com $10 \mathrm{~mL}$ de água esterilizada e espalhante adesivo (Tween 80) a $0,1 \%$. Após a agitação, foram feitas as diluições necessárias para a contagem do número de conídios, em microscópio óptico, com o auxílio de câmara de Neubauer.

A viabilidade dos conídios foi avaliada conforme metodologia descrita anteriormente, tendo-se estabelecido a percentagem de germinação. Foi realizado o cálculo do índice biológico (IB), para a classificação da toxicidade de agrotóxicos a fungos entomopatogênicos (Alves et al., 2007): tóxico
(0-41); moderadamente tóxico (42-66); e compatível $(>66)$. O cálculo do índice é realizado pela fórmula $\mathrm{IB}=[47(\mathrm{CV})+43(\mathrm{ESP})+10(\mathrm{GER})] / 100$, em que: $\mathrm{CV}$ é a percentagem do crescimento vegetativo da colônia após sete dias, em comparação à testemunha; ESP é a percentagem da esporulação das colônias após sete dias, em comparação à testemunha; GER é a percentagem de germinação dos conídios após 24 horas.

Para avaliação da compatibilidade de B. bassiana com os produtos fitossanitários, em condições de casa de vegetação, foram analisados os mesmos produtos utilizados no teste de compatibilidade em condições de laboratório (Tabela 1). Para tal, utilizaram-se mudas de Citrus sinensis, cultivar copa Pêra e cultivar porta-enxerto Limão-cravo de $40 \mathrm{~cm}$, que foram pulverizadas com $10 \mathrm{~mL}$ de calda de cada produto, com pulverizador costal. Cada produto representou um tratamento, e cada muda de citrus foi considerada uma repetição, no total de três repetições. O tratamento controle consistiu da aplicação do patógeno isoladamente, e a testemunha absoluta foi pulverizada apenas com água e espalhante adesivo (Tween 80) a $0,1 \%$. As mudas foram mantidas em vasos com volume de $3 \mathrm{~L}$, com substrato. A temperatura máxima da casa de vegetação variou de 28,5 a $36,6^{\circ} \mathrm{C}$, a mínima ficou entre 18,6 e $23,4^{\circ} \mathrm{C}$, a umidade relativa máxima variou entre 67,2 e $88,2 \%$ e a mínima ficou entre 24,4 e $56,5 \%$.

Os inseticidas e o extrato de nim foram aplicados nas concentrações recomendadas para a cultura, tendo-se aguardado duas horas para a secagem completa da calda sobre a planta. Em seguida, foi pulverizado o isolado de $B$. bassiana à $\mathrm{CL}_{90}$. Foram avaliados três tempos de exposição do entomopatógeno aos pesticidas: 2 horas, e 7 e 14 dias após a aplicação do produto. A avaliação das plantas foi realizada 24 horas após a aplicação do fungo. As plantas foram totalmente lavadas com $100 \mathrm{~mL}$

Tabela 1. Produtos utilizados na cultura de citros para o controle de Diaphorina citri, conforme lista de produtos registrados para a cultura, pelo Ministério da Agricultura, Pecuária e Abastecimento.

\begin{tabular}{|c|c|c|c|c|}
\hline Ingrediente ativo & Grupo químico & Característica & Dose recomendada & Modo de ação \\
\hline Malationa $1000 \mathrm{EC}$ & Organofosforado & Inseticida de contato e ingestão & $150 \mathrm{~mL}$ por $100 \mathrm{~L}$ de água & Inibidor de acetilcolinesterase \\
\hline Imidacloprido $200 \mathrm{SC}$ & Neonicotinoide & Inseticida sistêmico & $20 \mathrm{~mL}$ por $100 \mathrm{~L}$ de água & Antagonista de receptores \\
\hline Tiametoxam $250 \mathrm{WG}$ & Neonicotinoide & Inseticida sistêmico & 3 g por planta & nicotínicos da acetilcolina \\
\hline Piriproxifen 100 EC & Éter piridiloxipropílico & Inseticida de contato e translaminar & $6,25 \mathrm{~mL}$ por $100 \mathrm{~L}$ de água & Fisiológico juvenoide e $\mathrm{RC}^{(1)}$ \\
\hline Esfenvalerato $150 \mathrm{SC}$ & Piretroide & Inseticida de contato e ingestão & $12,5 \mathrm{~mL}$ por $100 \mathrm{~L}$ de água & Modulador de canais de sódio \\
\hline $\operatorname{Nim}(\text { Azadirachta indica })^{(2)}$ & - & - & $15 \mathrm{~mL}$ por $1 \mathrm{~L}$ de água & - \\
\hline
\end{tabular}

${ }^{(1)} \mathrm{RC}$, regulador de crescimento. ${ }^{(2)}$ Produto não registrado para a citricultura. 
de água destilada com espalhante adesivo (Tween 80 ) a $0,1 \%$, em potes de plástico, de $500 \mathrm{~mL}(11 \times 7,5 \mathrm{~cm})$, previamente esterilizados por 24 horas com luz UV. Em seguida, $0,1 \mathrm{~mL}$ dessa suspensão diluída $\left(\times 10^{-1}\right)$ foi inoculada, com auxilio de alça de Drigalsky, em quatro placas de Petri estéreis, por tratamento, que continham meio BDA acrescido de pentabiótico. As placas foram incubadas durante sete dias, a $25 \pm 1^{\circ} \mathrm{C}$ e fotófase de 12 horas. Após esse período, foi feita a contagem de colônias, para determinar o número de unidades formadoras de colônia (UFC) de B. bassiana .

Para se determinar a persistência de $B$. bassiana em plantas de citros, em condições de casa de vegetação, foram utilizadas mudas de $C$. sinensis, com a cultivar Pêra como copa e Limão-cravo, de $40 \mathrm{~cm}$, como porta-enxerto. As plantas foram pulverizadas com B. bassiana (IBCB 66) à $\mathrm{CL}_{90}$. Foram avaliados quatro tempos de persistência do entomopatógeno sobre a planta: 24 horas, 7 dias, 14 dias e 28 dias. Cada tempo representou um tratamento com 3 repetições. As mudas foram mantidas em casa de vegetação, nas mesmas condições de temperatura e umidade relativa descritas anteriormente. $\mathrm{O}$ tratamento controle foi pulverizado apenas com água e espalhante adesivo (Tween 80) a $0,1 \%$. Cada repetição foi constituída por uma planta. As plantas foram totalmente lavadas em $100 \mathrm{~mL}$ de água destilada e espalhante adesivo (Tween 80) a $0,1 \%$, em potes de plástico com volume de $500 \mathrm{~mL}$ (11 x 7,5 cm), previamente esterilizados com luz UV por 24 horas, e $0,1 \mathrm{~mL}$ dessa suspensão diluída $\left(\times 10^{-1}\right)$ foi espalhado, com auxilio de alça de Drigalsky, em quatro placas plásticas de Petri, por tratamento, com meio BDA acrescido de pentabiótico. As placas foram incubadas durante sete dias a $25 \pm 1^{\circ} \mathrm{C}$ e fotófase de 12 horas. Após esse período, foi feita a contagem de colônias.

Os dados obtidos nos experimentos foram submetidos ao teste $\mathrm{F}$, a $5 \%$ de probabilidade, e as médias foram comparadas pelo teste de Tukey, pelo programa Estat 2.0 (Sistema para Análises Estatísticas, Pólo Computacional, Departamento de Ciências Exatas, Unesp/FCAV, Campus de Jaboticabal, SP), em delineamento inteiramente casualizado. Para determinação da $\mathrm{CL}_{50 / 90}$ e $\mathrm{TL}_{50 / 90}$, foi realizada a análise Probit, com o programa Polo-PC (Leora, 1987). Para fins de análise estatística, os dados de contagem foram transformados em $(\mathrm{x}+10)^{0,5}$.

\section{Resultados e Discussão}

O fungo $B$. bassiana, IBCB 66, foi patogênico às ninfas de $D$. citri em todas as concentrações avaliadas. As maiores concentrações de inóculo causaram as maiores taxas de mortalidade (Figura 1).

Ninfas tratadas com $B$. bassiana tiveram comportamento semelhante ao das ninfas sadias, nos primeiros dias de avaliação. Com o tempo, as ninfas infectadas tornaram-se mais lentas, até não apresentar mobilidade, o que caracteriza o início do processo de morte. Houve alteração da coloração das ninfas, que variou de amarela para avermelhada (Figura 2), como resultado da ação da micotoxina oosporina (Alves, 1998).

A mortalidade foi confirmada pela visualização dos tegumentos encurvados, bem como pela não fixação das pernas e aparato bucal na planta hospedeira, tendose notado sintomas de desidratação, comprovados pelo arqueamento ventro-dorsal, e visível perda de volume dos corpos, ainda sem qualquer indício de esporulação do fungo. Os insetos que apresentaram colonização pelo patógeno e crescimento micelial foram considerados mortos pelo fungo (Figura 2).

O entomopatógeno mostrou-se um potencial agente de controle biológico de ninfas de $D$. citri, pois além de causar mortalidade acima de $40 \%$ em todas as concentrações, afetou a fisiologia do inseto e causou mortalidade durante o processo de ecdise.

A concentração que provocou a morte de $50 \%\left(\mathrm{CL}_{50}\right)$ dos insetos de uma população, após dez dias avaliação, foi a de $0,4 \times 10^{7}$ conídios $\mathrm{mL}^{-1}$. O menor tempo letal

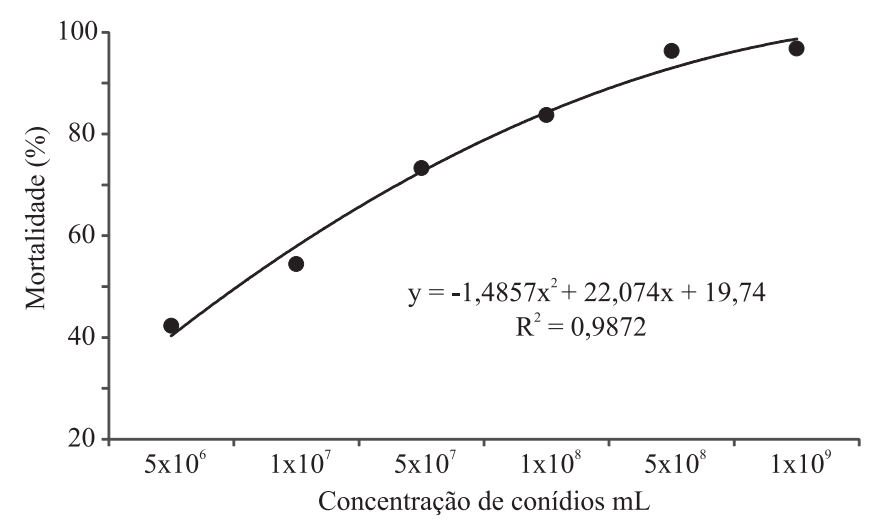

Figura 1. Mortalidade de Diaphorina citri, dez dias após a aplicação de diferentes concentrações do isolado IBCB 66 de Beauveria bassiana. 
$\left(\mathrm{TL}_{50}\right)$ foi de 5,7 dias, obtido com a maior concentração $\left(1 \times 10^{9}\right)$ (Tabela 2). Observou-se tendência de redução do tempo letal médio para as concentrações crescentes. Esse é um resultado frequente em estudos desta natureza (Fargues \& Rodrigues-Rueda, 1980).

Padulla \& Alves (2009) verificaram que o isolado Esalq-PL63 de $B$. bassiana causou alta mortalidade (em torno de $80 \%$ ) de ninfas de $D$. citri e calcularam uma $\mathrm{CL}_{50}$ de 2,3 x 107 , após 7 dias de incubação.

Rangel \& Correia (2003) avaliaram a virulência de Lecanicillium lecanii sobre o percevejo-de-renda da seringueira, Leptopharsa heveae Drake \& Poor (Heteroptera: Tingidae), e verificaram que este patógeno causou maior mortalidade à concentração de $2,4 \times 10^{7}$ conídios $\mathrm{mL}^{-1}$, com um $\mathrm{TL}_{50}$ de dois dias. Loureiro \& Moino Junior (2006) avaliaram a patogenicidade de fungos entomopatogênicos aos hemípteros Aphis gossypii Glover (Hemiptera: Aphididae) e Myzus persicae Sulzer (Hemiptera: Aphididae) e verificaram que os tempos letais diminuíram, à medida que as concentrações aumentaram, conforme o observado no presente trabalho.

Os produtos fitossanitários diferiram entre si quanto aos seus efeitos sobre o crescimento vegetativo do fungo, e todos diferiram da testemunha. A malationa foi o produto mais prejudicial ao crescimento de B. bassiana em meio de cultura (Tabela 3). Neves et al. (2001) avaliaram a compatibilidade dos fungos $B$. bassiana e $M$. anisopliae com inseticidas neonicotinoides, como acetamiprido, imidacloprido e tiametoxam, e verificaram redução significativa do crescimento vegetativo de $B$. bassiana, nos tratamentos com acetamiprido e tiametoxam nas doses recomendadas, conforme verificado no presente trabalho.
Quanto à esporulação, observou-se que os produtos não inibiram totalmente a esporulação do fungo e que o extrato de nim e o tiametoxam não apresentaram efeitos significativos sobre essa variável. Diversos autores observaram, em condições de laboratório, efeito positivo do inseticida tiametoxam sobre o crescimento vegetativo, esporulação e viabilidade de $B$. bassiana (Batista Filho et al., 2001; Neves et al., 2001; Almeida et al., 2003). Contudo, é provável que esse efeito dependa da concentração de tiametoxan utilizada. Cavalcanti et al. (2002) avaliaram o efeito dos produtos fitossanitários imidacloprido e tiametoxam sobre o fungo $B$. bassiana e observaram que esses inseticidas não afetaram o crescimento vegetativo e a esporulação, ao contrário do imidacloprido, que provocou redução dos parâmetros avaliados.

A presença dos agrotóxicos no meio de cultura afetou a viabilidade dos conídios, exceto o tiametoxam, que não diferiu da testemunha (Tabela 3). A malationa foi o produto de maior efeito, com $35,67 \%$ de redução da viabilidade. Quanto aos índices biológicos e a classificação dos produtos, o imidacloprido, nim, tiametoxam, esfenvalerato e piriproxifen foram classificados como moderadamente tóxicos (MT), e malationa foi considerado tóxico (T). Nenhum dos produtos testados foi classificado como compatível (Tabela 4).

A sobrevivência do isolado IBCB 66 de B. bassiana, em plantas de citros, em casa de vegetação, reduziu drasticamente ao longo do tempo de avaliação (Tabela 5).

Em casa de vegetação, todos os produtos se mostraram compatíveis com B. bassiana, e o número de unidades formadoras de colônias (UFC) nos

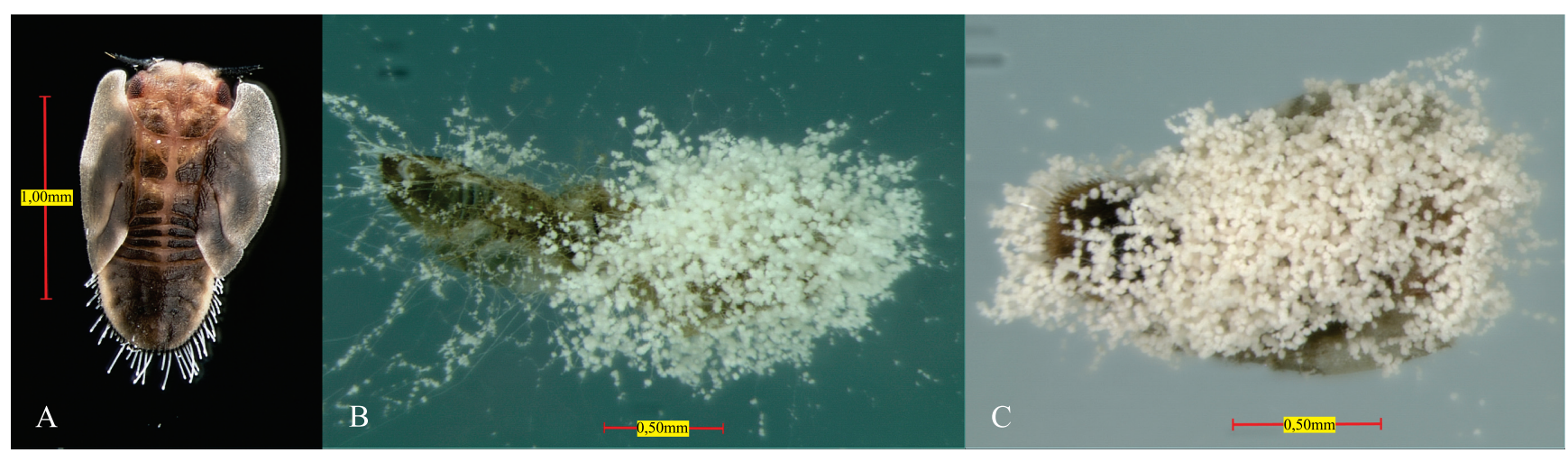

Figura 2. Mortalidade confirmada de Diaphorina citri, após a aplicação de diferentes concentrações de Beauveria bassiana. A, ninfa de D. citri morta, com coloração avermelhada característica da presença de micotoxina; B, ninfa de $D$. citri com mortalidade confirmada durante o processo de ecdise; e C, ninfa de D. citri com mortalidade confirmada. 
tratamentos não diferiu do observado na testemunha logo após a aplicação do fungo (Tabela 6). Gardner et al. (1984) verificaram que o número de colônias formadas imediatamente após os tratamentos foi mais alto; contudo, no presente trabalho, os valores de UFC logo após a aplicação foram menores do que os obtidos nos dois tempos seguintes de avaliação, 7 e 14 dias. Aos 7 e 14 dias, nenhum dos tratamentos diferiu da testemunha, o que mostra a compatibilidade do isolado de B. bassiana (IBCB 66) com os produtos analisados em casa de vegetação.

A ação dos produtos fitossanitários sobre o crescimento dos entomopatógenos pode variar em razão da natureza química dos produtos, do tempo de exposição e das condições ambientais (Tanada \& Kaya, 1992). Observa-se que a toxicidade dos produtos é maior nos ensaios com meio de cultura do que nos

Tabela 2. Tempos letais médios $\left(\mathrm{TL}_{50}\right)$, em dias, e equação de regressão linear, obtidos pela análise Probit da mortalidade de Diaphorina citri infectada com suspensão do fungo entomopatogênico Beauveria bassiana em diferentes concentrações de conídios.

\begin{tabular}{lcccccc}
\hline $\begin{array}{l}\text { Concentração } \\
\left(\text { conídios } \mathrm{mL}^{-1}\right)\end{array}$ & $\begin{array}{c}\mathrm{TL}_{50} \\
(\text { dias })\end{array}$ & $\begin{array}{c}\text { Intervalo de } \\
\text { confiança }\end{array}$ & & Equação & $\chi^{2}$ & $\mathrm{GL}$ \\
\hline $5 \times 10^{6}$ & 12,7 & $(11,2-15,7)$ & $\mathrm{y}=1,331+3,318 \mathrm{x}$ & 4,673 & 5 \\
$1 \times 10^{7}$ & 12,4 & $(10,3-16,8)$ & $\mathrm{y}=2,841+1,977 \mathrm{x}$ & 5,786 & 3 \\
$5 \times 10^{7}$ & 7,5 & $(7,0-8,0)$ & $\mathrm{y}=1,298+4,241 \mathrm{x}$ & 5,308 & 4 \\
$1 \times 10^{8}$ & 7,8 & $(7,5-8,1)$ & $\mathrm{y}=4,115+10,174 \mathrm{x}$ & 0,768 & 2 \\
$5 \times 10^{8}$ & 6,8 & $(6,5-7,0)$ & $\mathrm{y}=-3,360+10,063 \mathrm{x}$ & 1,719 & 3 \\
$1 \times 10^{9}$ & 5,7 & $(4,4-6,3)$ & $\mathrm{y}=-0,882+7,810 \mathrm{x}$ & 0,868 & 2 \\
\hline
\end{tabular}

$\chi^{2}$, qui-quadrado.

Tabela 3. Valores médios do crescimento vegetativo, esporulação e viabilidade de colônias do isolado IBCB 66 de Beauveria bassiana, em presença de produtos químicos utilizados na citricultura, em condições de laboratório ${ }^{(1)}$.

\begin{tabular}{lccc}
\hline Tratamento & Diâmetro $(\mathrm{cm})$ & Conídios $\left(\times 10^{7}\right)^{(2)}$ & Viabilidade (\%) \\
\cline { 2 - 4 } Testemunha & $1,53 \mathrm{a}$ & $2,5 \mathrm{a}$ & $95,33 \mathrm{a}$ \\
Imidacloprido 200 SC & $1,19 \mathrm{~b}$ & $0,7 \mathrm{~b}$ & $90,33 \mathrm{~b}$ \\
Nim & $1,13 \mathrm{~b}$ & $0,9 \mathrm{ab}$ & $51,67 \mathrm{~b}$ \\
Tiametoxam 250 WG & $1,22 \mathrm{~b}$ & $0,9 \mathrm{ab}$ & $94,67 \mathrm{a}$ \\
Esfenvalerato 150 SC & $1,16 \mathrm{~b}$ & $0,4 \mathrm{~b}$ & $91,33 \mathrm{~b}$ \\
Malationa 1000 EC & $0,48 \mathrm{c}$ & $0,5 \mathrm{~b}$ & $35,67 \mathrm{~b}$ \\
Piriproxifen 100 EC & $1,27 \mathrm{~b}$ & $0,3 \mathrm{~b}$ & $90,33 \mathrm{~b}$ \\
\hline Teste F & $30,29 * *$ & $4,24 * *$ & $2.325,88^{* *}$ \\
CV (\%) & 12,45 & 36,92 & 1,11 \\
\hline
\end{tabular}

${ }^{(1)}$ Médias seguidas de letras iguais não diferem entre si, pelo teste de Tukey, a $5 \%$ de probabilidade. ${ }^{(2)}$ Dados originais. $* *$ Significativo a $1 \%$ de probabilidade pelo teste $\mathrm{F}$. ensaios em casa de vegetação. Isto ocorre porque a homogeneidade do meio facilita a distribuição do produto e promove ação rápida e efetiva sobre o fungo, o que provavelmente não ocorre no ambiente. No meio de cultura, há contato permanente do patógeno com a elevada concentração do produto e não ocorre degradação do produto químico pela incidência de radiação solar. Batista Filho et al. (2001) avaliaram o número médio de UFC de $M$. anisopliae e $B$. bassiana, em folhas de feijãosubmetidas ao inseticida tiametoxam, nos tempos de 24, 48 e 72 horas após a pulverização. Os autores verificaram que o inseticida não interferiu no número médio de UFC, independentemente do microrganismo estudado.

Tabela 4. Valores do índice biológico (IB) ${ }^{(1)}$ e classificação de produtos químicos utilizados na citricultura quanto à toxicidade a Beauveria bassiana, em condições de laboratório.

\begin{tabular}{lccc}
\hline Tratamento & Índice biológico & & Classificação \\
\cline { 1 - 2 } Imidacloprido 200 SC & 58 & & Moderadamente tóxico \\
Nim & 56 & & Moderadamente tóxico \\
Tiametoxam 250 WG & 62 & Moderadamente tóxico \\
Esfenvalerato 150 SC & 52 & Moderadamente tóxico \\
Malationa 1000 EC & 27 & Tóxico \\
Piriproxifen 100 EC & 53 & Moderadamente tóxico \\
\hline
\end{tabular}

${ }^{(1)}$ Calculado segundo a fórmula IB $=[47(\mathrm{CV})+43(\mathrm{ESP})+10(\mathrm{GER})] / 100$, em que: CV é a percentagem de crescimento vegetativo da colônia, após sete dias, em comparação à testemunha; ESP é a percentagem da esporulação das colônias, após sete dias, em comparação à testemunha; e GER é a percentagem de germinação dos conídios, após 24 horas.

Tabela 5. Sobrevivência do isolado IBCB 66 de Beauveria bassiana, medida pelo número de unidades formadoras de colônia após diferentes tempos de contato com os produtos químicos em plantas de citrus, em casa de vegetação ${ }^{(1)}$.

\begin{tabular}{lccr}
\hline Tratamento & $\begin{array}{c}\text { Imediatamente após } \\
\text { a aplicação }\end{array}$ & 7 dias & 14 dias \\
\hline Testemunha (água) & $0,25 \mathrm{~b}$ & $0,00 \mathrm{c}$ & $0,00 \mathrm{~b}$ \\
Testemunha B. bassiana & $290,25 \mathrm{a}$ & $635,41 \mathrm{ab}$ & $806,00 \mathrm{a}$ \\
Imidacloprido 200 SC & $281,08 \mathrm{a}$ & $504,41 \mathrm{~b}$ & $545,08 \mathrm{a}$ \\
Nim & $290,66 \mathrm{a}$ & $728,50 \mathrm{ab}$ & $609,17 \mathrm{a}$ \\
Tiametoxam 250 WG & $301,08 \mathrm{a}$ & $605,41 \mathrm{ab}$ & $522,92 \mathrm{a}$ \\
Esfenvalerato 150 SC & $298,00 \mathrm{a}$ & $689,33 \mathrm{ab}$ & $487,42 \mathrm{a}$ \\
Malationa 1000 EC & $306,75 \mathrm{a}$ & $796,49 \mathrm{a}$ & $606,08 \mathrm{a}$ \\
Piriproxifen 100 EC & $238,91 \mathrm{a}$ & $871,08 \mathrm{a}$ & $561,33 \mathrm{a}$ \\
\hline Teste F & $26,61^{* *}$ & $20,98^{* *}$ & $37,60^{* *}$ \\
CV (\%) & 15,98 & 19,46 & 12,84 \\
\hline
\end{tabular}

${ }^{(1)}$ Médias seguidas de letras iguais, nas colunas, não diferem entre si, pelo teste de Tukey, a $5 \%$ de probabilidade **Significativo a $1 \%$ de probabilidade, pelo teste $\mathrm{F}$. 
Tabela 6. Persistência do isolado IBCB 66 de Beauveria bassiana, em plantas de citrus, medida pelo número de unidades formadoras de colônia após diferentes tempos da aplicação do fungo às plantas, em condições de campo ${ }^{(1)}$.

\begin{tabular}{lrccc}
\hline Tratamento & 24 horas & 7 dias & 14 dias & 28 dias \\
\hline Testemunha - água & $0,00 \mathrm{~b}$ & $0,00 \mathrm{~b}$ & $0,00 \mathrm{~b}$ & $0,00 \mathrm{~b}$ \\
B. bassiana & $269,83 \mathrm{a}$ & $13,17 \mathrm{a}$ & $7,17 \mathrm{a}$ & $1,00 \mathrm{a}$ \\
\hline Teste F & $79,42^{* *}$ & $11,68^{*}$ & $11,47^{*}$ & $4,79 \mathrm{~ns}$ \\
CV $(\%)$ & 18,69 & 14,50 & 9,50 & 2,82 \\
\hline
\end{tabular}

(1)Médias seguidas de letras iguais, nas colunas, não diferem entre si, pelo teste de Tukey, a $5 \%$ de probabilidade. ${ }^{* * S i g n i f i c a t i v o ~ a ~} 1 \%$ de probabilidade, pelo teste $\mathrm{F}$.

O estudo de compatibilidade, para simular a aplicação real a campo, evidenciou que os produtos não afetaram a sobrevivência do inóculo nas plantas, quando aplicados anteriormente a $B$. bassiana. $\mathrm{O}$ estabelecimento do tempo mais adequado para a aplicação é importante para programas de controle biológico, manejo integrado de pragas ou produção integrada de frutas. Loria et al. (1983) relataram que o tempo de aplicação pode ser um aspecto importante na sobrevivência de conídios de $B$. bassiana no campo. Esses autores observaram que a aplicação do patógeno 10 e 28 horas após a aplicação dos fungicidas metiram, metalaxil e clorotalonil não causou mortalidade de conídios, pois foram constatadas UFC do fungo, apesar da diferença entre os produtos analisados.

\section{Conclusões}

1. O isolado IBCB 66 de Beauveria bassiana reduz a sobrevivência de ninfas de Diaphorina citri.

2. As maiores concentrações do inóculo de $B$. bassiana aumentam a mortalidade de $D$. citri e diminuem o tempo letal.

3. Malationa, imidacloprido, tiametoxam, piriproxifen, esfenvalerato e extrato de nim reduzem o crescimento do isolado IBCB 66 de B. bassiana, em condições de laboratório, mas não afetam a sobrevivência do fungo nas plantas de citrus, em casa de vegetação.

4. A malationa é classificada como tóxica a B. bassiana, e imidacloprido, tiametoxam, piriproxifen, esfenvalerato e extrato de nim, como moderadamente tóxicos.

\section{Referências}

ALEMÁN, J.; BAÑOS, H.; RAVELO, J. Diaphorina citri y la enfermedad huanglongbing: una combinación destructiva para la producción citrícola. Revista de Protección Vegetal, v.22, p.154-165, 2007.

ALMEIDA, J.E.M.; BATISTA FILHO, A.; LAMAS, C.; LEITE, L.G.; TRAMA, M.; SANO, A.H. Avaliação da compatibilidade de defensivos agrícolas na conservação de microrganismos entomopatogênicos no manejo de pragas do cafeeiro. Arquivos do Instituto Biológico, v.70, p.79-84, 2003.

ALVES, S.B. Fungos entomopatogênicos. In.: ALVES, S.B. (Ed.). Controle microbiano de insetos. 2.ed. Piracicaba: FEALQ, 1998. p.289-381.

ALVES, S.B.; HADDAD, M.L.; FAION, M.; BAPTISTA, G.C.; ROSSI-ZALAF, L.S. Novo índice biológico para classificação da toxicidade de agrotóxicos para fungos entomopatogênicos. In: SIMPÓSIO DE CONTROLE BIOLÓGICO, 10., 2007, Brasília. Resumos. Brasília: Embrapa, 2007. CD-ROM.

AVERY, P.B.; HUNTER, W.B.; HALL, D.G.; JACKSON, M.A.; POWELL, C.A.; ROGERS, M.E. Diaphorina citri (Hemiptera: Psyllidae) infection and dissemination of the entomopathogenic fungus Isaria fumosorosea (Hypocreales: Cordycipitaceae) under laboratory conditions. Florida Entomologist, v.92, p.608-618, 2009.

BATISTA FILHO, A.; ALMEIDA, J.E.M.; LANAS, C. Effect of thiametoxam on entomopathogenic microorganisms. Neotropical Entomology, v.30, p.437-447, 2001.

BOINA, D.R.; ONAGBOLA, E.O.; SALYANI, M.; STELINSKI, L.L. Antifeedant and sublethal effects of imidacloprid on Asian citrus psyllid, Diaphorina citri. Pest Management Science, v.65, p.870-877, 2009.

BOVÉ, J.M. Huanglongbing: a destructive, newly-emerging, century-old diseasse of citrus. Journal of Plant Pathology, v.88, p.7-37, 2006.

CAVALCANTI, R.S.; MOINO JUNIOR, A.; SOUZA, G.C.; ARNOSTI, A. Efeito dos produtos fitossanitários fenpropatrina, imidaclopride, iprodione e tiametoxam sobre o desenvolvimento do fungo Beauveria bassiana (Bals.) Vuill. Arquivos do Instituto Biológico, v.69, p.17-22, 2002.

COCCO, A.; HOY, M.A. Toxicity of organosilicone adjuvants and selected pesticides to the Asian citrus psyllid (Hemiptera: Psyllidae) and its parasitoid Tamarixia radiata (Hymenoptera: Eulophidae). Florida Entomologist, v.91, p.610-620, 2008.

FARGUES, J.; RODRIGUES-RUEDA, D. Sensibilité des larves de Spodoptera litoralis (Lepidoptera: Noctuidae) aux hyphomycètes entomopathogènes Nomureae rileyi et Paecilomyces fumosoroseus. Entomophaga, v.25, p.43-54, 1980 .

GÓMEZ-TORRES, M.L. Estudos bioecológicos de Tamarixia radiata (Waterston, 1922) (Hymenoptera: Eulophidae) para o controle de Diaphorina citri Kuwayama, 1907 (Hemiptera: Psyllidae). 2009. 138p. Tese (Doutorado) - Escola Superior de Agricultura Luiz de Queiroz, Piracicaba.

LORIA, R.; GALAINI S.; ROBERTS, D.W. Survival of inoculum of the entomopathogenic fungus Beauveria bassiana as influenced by fungicides. Environmental Entomology, v.12, p.17241726, 1983. 
LOUREIRO, E. de S.; MOINO JUNIOR, A. Patogenicidade de fungos hifomicetos aos pulgões Aphis gossypii (Glover) e Myzus persicae (Sulzer) (Hemiptera: Aphididae). Neotropical Entomology, v.35, p.660-665, 2006.

MARQUES, R.P.; MONTEIRO,A.C.;PEREIRA, G.T.Crescimento, esporulação e viabilidade de fungos entomopatogênicos em meios contendo diferentes concentrações de óleo de nim (Azadirachta indica). Ciência Rural, v.34, p.1675-1680, 2004.

MCCOY, C.W.; STOREY, G.K.; TIGANO-MILANI, M.S. Environmental factors affecting entomopathogenic fungi in the soil. Pesquisa Agropecuária Brasileira, v.27, p.107-111, 1992.

NAVA, D.E.; TORRES, M.L.G.; RODRIGUES, M.D.L.; BENTO, J.M.S.; PARRA, J.R.P. Biology of Diaphorina citri (Hem., Psyllidae) on different hosts and at different temperatures. Journal of Applied Entomology, v.131, p.709-715, 2007.

NEVES, P.M.O.J.; HIROSE, E.; TCHUJO, P.T.; MOINO JUNIOR, A. Compatibility of entomopathogenic fungi with neonicotinoids insecticides. Neotropical Entomology, v.30, p.263-268, 2001.

PADULLA, L.F.L.; ALVES, S.B. Suscetibilidade de ninfas de Diaphorina citri a fungos entomopatogênicos. Arquivos do Instituto Biológico, v.76, p.297-302, 2009.

PEREIRA, F.F.; BARROS, R.; PRATISSOLI, D.; PARRA, J.R.P. Biologia e exigências térmicas de Trichogramma pretiosum Riley e T. exiguum Pinto \& Platner (Hymenoptera: Trichogrammatidae), criados em ovos de Plutella xylostella (L.) (Lepidoptera: Plutellidae). Neotropical Entomology, v.33, p.231-236, 2004.

RANGEL, D.E.N.; CORREIA, A. do C.B. Virulência de Aphanocladium album (Preuss) Gams e Verticillium lecanii (Zimm.) Viégas (Deuteromycotina: Hyphomycetes) para o percevejo-de-renda da seringueira, Leptopharsa heveae (Drake \& Poor) (Hemiptera: Tingidae). Ciência e Agrotecnologia, v.27, p.1636-1642, 2003. Edição especial.

SANCHES, A.L.; FELIPPE, M.R.; CARMO, A.U.; RUGNO, G.R.; YAMAMOTO, P.T. Eficiência de inseticidas sistêmicos, aplicados em mudas cítricas, em pré-plantio, no controle de Diaphorina citri (Kuwayama) (Hemiptera: Psyllidae). BioAssay, v.4, p.1-7, 2009.

SOUZA, J.C.; REIS, P.R. Controle da traça-do-tomateiro em Minas Gerais. Pesquisa Agropecuária Brasileira, v.21, p.343-354, 1986.

TANADA, Y.; KAYA, H.K. Fungal infections. In: TANADA, Y.; KAYA, H.K. (Ed.). Insect pathology. San Diego: Academic Press, 1992. p.318-387.

TSAI, J.H.; LIU, Y.H. Biology of Diaphorina citri (Homoptera: Psyllidae) on four host plants. Journal of Economic Entomology, v.93, p.1721-1725, 2000.

YAMAMOTO, P.T.; FELIPPE, M.R.; SANCHES, A.L.; COELHO, J.H.C.; GARIM, L.F.; XIMENES, N.L. Eficácia de inseticidas para o manejo de Diaphorina citri Kuwayama (Hemiptera: Psyllidae) em citros. BioAssay, v.4, p.1-9, 2009.

Recebido em 24 de abril de 2012 e aprovado em 4 de novembro de 2012 